\title{
EFEITO DO COMPOSTO DE LIXO URBANO EM UM SOLO ARENOSO E EM PLANTAS DE SORGO(1)
}

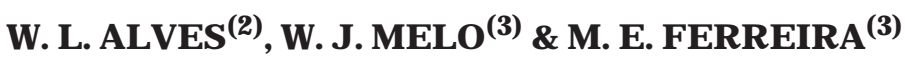

\begin{abstract}
RESUMO
Estudou-se o efeito do composto de lixo urbano na fertilidade e nos teores totais e extraídos com solução de DTPA 0,005 mol L-1 pH 7,3 dos elementos $\mathrm{Fe}$, Mn, Zn e Ni em um Podzólico Vermelho-Amarelo textura arenosa, em casa de vegetação. E mpregou-se sorgo como planta-teste, avaliando-se perfilhamento, produção de matéria seca e quantidade acumulada dos elementos $\mathrm{P}, \mathrm{K}, \mathrm{Ca}, \mathrm{Mg}$, $\mathrm{Fe}, \mathrm{Mn}, \mathrm{Zn}$ e $\mathrm{Ni}$ em função de doses de composto equivalentes a $0,12,5,25,50$ e $100 \mathrm{t} \mathrm{ha}^{-1}$, na ausência e na presença de adubação mineral. 0 composto aumentou a produção de matéria seca do sorgo na ausência de adubação mineral; esta, contudo, promoveu maior aumento de produção e perfilhamento das plantas. $\mathrm{Na}$ parte aérea de sorgo, não foi detectado $\mathrm{Ni}$, enquanto as quantidades acumuladas de Fe, Mn e Zn relacionaram-se com os teores no solo extraídos com DTPA. A aplicação do composto de lixo promoveu aumento dos valores de pH, matéria orgânica, bases trocáveis, P resina e CTC do solo, reduzindo a acidez potencial $(\mathrm{H}+\mathrm{Al})$. Os teores totais de $\mathrm{Fe}, \mathrm{Mn}$ e $\mathrm{Zn}$ no solo aumentaram com as doses do composto, enquanto o de $\mathrm{Ni}$ aumentou somente na ausência de adubação mineral. Os teores de $\mathrm{Zn}$ e Ni no solo, extraídos com DTPA, aumentaram com as doses do composto tanto na ausência como na presença de adubação mineral; já os de Mn eF e diminuíram na presença de adubação mineral e não foram alterados na sua ausência. Apesar de não ter ocorrido acúmulo dos metais pesados no tecido vegetal, houve incremento no solo de al guns dos elementos; portanto, devese atentar para aplicações sucessivas de composto de lixo domiciliar.
\end{abstract}

Termos de indexação: adubação orgânica, DTPA, metais pesados, acidez do solo.

(1) Parte da Tese de Mestrado em Agronomia do primeiro autor apresentada à FCAV/UNESP. Recebido para publicação em março de 1997 e aprovado em abril de 1999.

(2) Doutorando do Departamento deSolos eAdubos, FCAV/UNESP Campus deJ aboticabal. Via deAcesso Prof. Paulo Donato Castellane, s/n, CEP 14870-000 J aboticabal (SP). Bolsista do CNPq. E. mail: william@fcav.unesp.br.

(3) Professor Titular da FCAV/UNESP. 


\title{
SUMMARY: URBAN WASTE COMPOST EFFECTS ON SANDY SOIL AND SORGHUM PLANTS
}

\begin{abstract}
A greenhouse experiment was carried out to evaluatethe effect of urban wastecompost on soil fertility and on total and extracted contents (DTPA $0.005 \mathrm{~mol} \mathrm{~L}^{-1} \mathrm{pH} 7.3$ ) of the elements $\mathrm{Fe}, \mathrm{Mn}, \mathrm{Zn}$ and $\mathrm{Ni}$ in a sandy Red-Yellow Podzolic soil (Ultisol). Theyield of dry matter of Sorghum bicol or plants and the content of $\mathrm{P}, \mathrm{K}, \mathrm{Ca}, \mathrm{Mg}, \mathrm{Fe}, \mathrm{Mn}, \mathrm{Zn}$ and $\mathrm{Ni}$ were eval uated in plant tops as a function of compost rates of $0,12.5,25,50$ and $100 \mathrm{t} \mathrm{ha}^{-1}$, in the absence and presence of mineral fertilizers. Dry matter yield increased with the compost rates only in the absence of mineral fertilization. $\mathrm{Ni}$ was not detected in the plant tops of sorghum, whereas $\mathrm{Fe}, \mathrm{Mn}$ and $\mathrm{Zn}$ contents werere ated with theDTPA-extracted contents of these elements in the soil. The application of urban waste compost increased $\mathrm{pH}$, organic matter, exchangeablebases, P-resin and CEC values of thesoi I, reducing the potential acidity $(\mathrm{H}+\mathrm{Al})$. Thetotal contents of $\mathrm{Fe}, \mathrm{Mn}$ and $\mathrm{Zn}$ in the soil increased with the compost rates, whereas thetotal $\mathrm{Ni}$ content increased only in theabsence of mineral fertilization. Thecontents of $\mathrm{Zn}$ and $\mathrm{Ni}$ in thesoil, extracted with DTPA, increased with the compost rates both in the absenceand in thepresence of mineral fertilization; $\mathrm{Mn}$ and Fecontents, however, decreased in the presence of mineral fertilization and were not affected in its absence. Although accumulation of theheavy metals in theplants was not detected, thecontent of someel ements in thesoil increased; therefore, attention should begiven to the possi bility of soil contamination when conducting successi ve applications of urban waste compost.
\end{abstract}

Index terms: organic fertilization, DTPA, heavy metals, MSW, soil aci dity.

\section{INTRODUÇÃO}

Um dos grandes problemas ambientais da atualidade diz respeito à disposição de resíduos sólidos de origem domiciliar. Tais resíduos, quando dispostos no solo sem tratamento e em grandes quantidades, provocam graves problemas de contaminação ambiental (Kiehl, 1985; J ardim et al., 1995; Alves, 1998). Os resíduos domiciliares são constituídos por 50 a $60 \%$ de material orgânico, e a sua reciclagem, por meio do processo de compostagem e posterior aplicação agrícola, é uma maneira eficiente de reduzir o volume de material destinado aos aterros sanitários (Hernandez et al., 1992; Grossi, 1993). O composto obtido do lixo é um adubo orgânico com boas características agronômicas, atuando na mel horia de propriedades físicas, químicas e biológicas do solo (Kiehl, 1985; Nakagawa, 1992).

Hortenstine \& Rothwell (1973), avaliando os efeitos fitotóxicos dos micronutrientes $B, M n$ e $Z n$ presentes no composto, revelaram que as quantidades absorvidas desses elementos não causava preocupação de contaminação, mesmo na dose mais el evada de 64 t ha-1. Cabrera et al. (1989), comparando a aplicação de $14 \mathrm{t}$ ha-1 de composto de lixo com $500 \mathrm{~kg} \mathrm{ha-1}$ de um fertilizante N-P-K, na fórmula 15-15-15, para a cultura do sorgo por dois ciclos, observaram quea dose utilizada foi suficiente para manter níveis adequados de $\mathrm{P}$ e $\mathrm{K}$ no solo e não promoveu diferença de produção, quando comparada com a do fertilizante mineral
$\mathrm{N}-\mathrm{P}-\mathrm{K}$ sozinho. Os autores alertaram para possíveis problemas com excesso de Cu e Zn, se doses maiores do composto forem utilizadas ou aplicações sucessivas forem feitas. Costa et al . (1994) estudaram, em casa de vegetação, o efeito da aplicação de composto de lixona produção de al facee noteor de metais pesados na planta eobservaram que, em sol os argilosos, houve aumento de produção de matéria seca e, em solos arenosos, efeito depressivo na produção de alface. Segundo os referidos autores, verificou-se aumento significativo do teor de $\mathrm{Zn}, \mathrm{Cu}, \mathrm{Cd}$ e $\mathrm{Pb}$ nas fol has, apenas o Cu atingiu teores considerados fitotóxicos; o teor de $\mathrm{Pb}$ atingiu o limite de tolerância em alimentos fixado pela legislação brasileira.

Collier et al. (1997) avaliaram amostras desolo, de fol has e de frutos degoiabeira deáreas que receberam adubação com composto de lixo (40 t ha-1 ano-1), por 11 anos, bem como deáreas que receberam composto apenas no plantio das mudas (40 t ha-1 em aplicação única) e daquelas que não receberam composto. Os autores concluíram que, dentro dosistema de manejo do solo avaliado, não ocorreu acúmulo de metais pesados nas fol has ou frutos de goiabeira acima dos níveis encontrados na literatura; houveenriquecimento de metais pesados no solo das duas áreas que receberam composto, embora não atingindo níveis críticos citados pela literatura. Outros autores relataram efeitos na fertilidade do solo como elevação do pH (Coker \& Mathews, 1983; Simeoni et al., 1984; Pereira Neto, 1987; Hernando et al., 1989) e aumento de bases trocáveis (Costa et al., 1992). Apesar do grande número de trabal hos com 
aplicação de composto de lixo real izados no Brasil e no mundo, existem dúvidas quanto à sua eficiência agronômica e possibilidade de contaminação por metais pesados, principal menteno Brasil, ondeainda são poucos os trabal hos nesta linha de pesquisa.

Este trabal ho teve como objetivo avaliar, em casa de vegetação, os efeitos da aplicação do composto de lixo urbano na fertilidade de um solo arenoso e no desenvol vimento e absorção de nutrientes e metais pesados por plantas de sorgo.

\section{MATE RIAL E MÉTODOS}

O experimento foi realizado em casa de vegetação da Estação Experimental da CETESB de Novo Horizonte (SP), no período de julho a novembro de 1995.

Utilizaram-se amostras de um Podzólico Vermelho-Amarelo textura arenosa $\left(830 \mathrm{~g} \mathrm{~kg}^{-1}\right.$ de areia e $130 \mathrm{~g} \mathrm{~kg}^{-1}$ de argila), coletadas na camada de $0-20 \mathrm{~cm}$ de profundidade, no município de Novo Horizonte (SP), com as seguintes propriedades químicas: $\mathrm{P}$ (resina) $=2 \mathrm{mg} \mathrm{dm}^{-3}$, matéria orgânica $=13 \mathrm{~g} \mathrm{dm}^{-3}, \mathrm{pH} \mathrm{em} \mathrm{CaCl} 2=4,6, \mathrm{~K}=2,3 \mathrm{mmol}_{\mathrm{c}} \mathrm{dm}^{-3}$, $\mathrm{Ca}=15 \mathrm{mmol}_{\mathrm{c}} \mathrm{dm}^{-3}, \mathrm{Mg}=3 \mathrm{mmol}_{\mathrm{c}} \mathrm{dm}^{-3}, \mathrm{H}+\mathrm{Al}=$ $28 \mathrm{mmol}_{\mathrm{c}} \mathrm{dm}^{-3}$, soma de bases $=20 \mathrm{mmol}_{\mathrm{c}} \mathrm{dm}^{-3}, \mathrm{CTC}$ $=48 \mathrm{mmol}_{\mathrm{c}} \mathrm{dm}^{-3}$ e saturação por bases $=42 \%$, de acordo com o método descrito em Raij et al. (1987). Real izou-setambém a determinação dos teores totais de micronutrientes e metais pesados, segundo o método descrito em Baker \& Amacher (1982), obtendo-se os seguintes resultados, em $\mathrm{mg} \mathrm{kg}^{-1}: \mathrm{Zn}$ $=10, \mathrm{Mn}=62, \mathrm{Fe}=13.650, \mathrm{Ni}=$ não detectado; $\mathrm{Cd}=$ não detectado e $\mathrm{Pb}=$ não detectado.

O composto utilizado foi obtido na usina piloto decompostagem delixo urbano da CETESB, de Novo Horizonte (SP), produzido ao final de um processo de 120 dias de compostagem pelo sistema de leiras a céu aberto, com revolvimentos periódicos (Alves, 1998). Análiserealizada segundo método descrito por Kiehl (1985) revelou um pH em $\mathrm{CaCl}_{2}$ de 8,07 e os seguintes resultados na base seca: $\mathrm{N}=7 \mathrm{~g} \mathrm{~kg}^{-1} ; \mathrm{P}=$ $5 \mathrm{~g} \mathrm{~kg}^{-1} ; \mathrm{K}=0,7 \mathrm{~g} \mathrm{~kg}^{-1} ; \mathrm{Ca}=3,6 \mathrm{~g} \mathrm{~kg}^{-1}, \mathrm{Mg}=0,3 \mathrm{~g} \mathrm{~kg}^{-1}$, matéria orgânica $=300 \mathrm{~g} \mathrm{dm}^{-3}$ e relação $\mathrm{C} / \mathrm{N}=12 / 1$. Determinaram-se os teores de micronutrientes e metais pesados por meio do método de Baker \& Amacher (1982), obtendo-se os seguintes resultados em $\mathrm{mg} \mathrm{kg}^{-1}$ na base seca: $\mathrm{Zn}=310, \mathrm{Mn}=234, \mathrm{Fe}=$ 27100 e Ni $=12$.

Empregou-se um esquema fatorial $2 \times 5 \mathrm{com}$ 10 tratamentos, repetidos quatro vezes em delineamento inteiramente casualizado, totalizando 40 vasos. Os tratamentos foram constituídos pela combinação dos fatores adubação mineral (presença e ausência) e composto de lixo (nas doses equival entes a $0,12,5,25,50$ e 100 t ha-1 do material seco ao ar). A adubação mineral correspondeu, em $\mathrm{mg} \mathrm{dm}^{-3}$, a 150 de N, 200 deP, 150 deK, 30 deS, 1 de $B, 15$ de $F$ e, 15 de $M n, 0,1$ de Mo e 4 deZn. Os diversos produtos utilizados, suas respectivas concentrações nas soluções e quanti dades aplicadas por vaso foram: $\left(\mathrm{NH}_{4}\right)_{2} \mathrm{SO}_{4} / 16,4 \mathrm{~g} \mathrm{~L}-1 / 60 \mathrm{~mL} ; \mathrm{NH}_{4} \mathrm{H}_{2} \mathrm{PO}_{4} / 51,93 \mathrm{~g} \mathrm{~L}-1 /$ $50 \mathrm{~mL} ; \mathrm{KCl} / 11,14 \mathrm{~g} \mathrm{~L}-1 / 90 \mathrm{~mL} ; \mathrm{H}_{3} \mathrm{BO}_{3} / 0,657 \mathrm{~g} \mathrm{~L}^{-1 /}$ $30 \mathrm{~mL}$; Fe EDTA/6,3 g L-1/30 mL; $\mathrm{MnCl}_{2} \cdot 4 \mathrm{H}_{2} \mathrm{O} /$

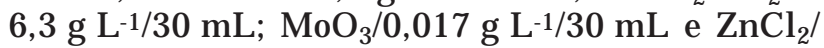
0,976 g L-1/30 mL.

Na instalação do experimento, utilizaram-se, como vasos, tubos de PVC de $15 \mathrm{~cm}$ de diâmetro e $40 \mathrm{~cm}$ dealtura, contendo, a $10 \mathrm{~cm}$ da base, uma tela de plástico com abertura de malha de $1 \mathrm{~mm}$, coberta por um disco de papel de filtro Whatmam no 1 para sustentação do solo.

Amostras de solo (3,5 dm-3), secas e passadas em peneira de $4 \mathrm{~mm}$, receberam cal cário dol omítico para elevar a saturação por bases a $70 \%$ e, em seguida, adubo mineral e composto de lixo igual mente seco e passado em peneira de $4 \mathrm{~mm}$; após homogeneização, as amostras foram colocadas nos tubos, resultando em coluna de $28 \mathrm{~cm}$ de altura. Adicionou-se água desionizada em quantidade suficiente para el evar a umidade do solo a $70 \%$ da capacidade de retenção de água, ficando nestas condições por 40 dias para incubação; nesse período, a umi dadefoi mantida por meio de pesagens e reposição de água diárias. Semearam-se cinco sementes de sorgo [Sorghum bicol or (L.) Moench, variedade Ag 405] na profundidade de $1 \mathrm{~cm}$ e, 20 dias após, foi feito desbaste, deixando-se uma planta por vaso. Aos 97 dias da semeadura, o número de perfilhos foi determi nado ea parteaérea das plantas foi col etada, lavada uma vez em água de torneira, uma vez em água de torneira com detergente neutro a 0,1\% e mais duas vezes em água desionizada; em seguida, foi seca em estufa, a aproximadamente $65^{\circ} \mathrm{C}$, pesada, moída e submetida à determinação da quantidade acumulada de: $\mathrm{P}$, em extrato nítrico-perclórico, por colorimetria; $\mathrm{K}, \mathrm{Ca}, \mathrm{Mg}$, no mesmo extrato, por espectrofotometria de absor ção atômica (Sarruge \& Haag, 1974), e Fe, Mn, Zn e Ni, no extrato nítricoperclórico, por espectrofotometria de absorção atômica (Baker \& Amacher, 1982).

Amostras de terra de cada vaso foram col etadas para determinação dos parâmetros de fertilidade, P-resina, matéria orgânica, pH, $\mathrm{K}, \mathrm{Ca}, \mathrm{MgeH}+\mathrm{Al}$, segundo método de Raij et al. (1987). Determinaram$\mathrm{se}$, ainda, os teores totais dos el ementos $\mathrm{Fe}, \mathrm{Mn}, \mathrm{Zn}$ e $\mathrm{Ni}$ no extrato nítrico-perclórico por espectrofotometria de absorção atômica (Baker $\&$ Amacher, 1982), bem como os teores dos mesmos el ementos, extraídos em solução deDTPA 0,005 mol L-1 pH 7,3, conforme descrito por Lindsay \& Norwell (1978).

Os resultados foram submetidos à análise de variância em esquema fatorial, e os efeitos de doses foram avaliados por meio de regressão pol inomial. 


\section{RESULTADOSE DISCUSSÃO}

\section{Fertilidade do solo}

Os teores de P, K, Ca e Mg sofreram aumentos significativos em função da dose do composto, tanto na ausência como na presença de adubação mineral (Figura 1). No caso do Mg, contudo, não houve inter ação de doses de composto e adubação mineral . Esses resultados corroboram os obtidos por Hortenstine\& Rothwell (1972), que observaram, em solos arenosos de baixa fertilidade, aumento nos teores de Ca, Mg e K trocáveis após a aplicação de 35 e 70 t ha-1 do composto.
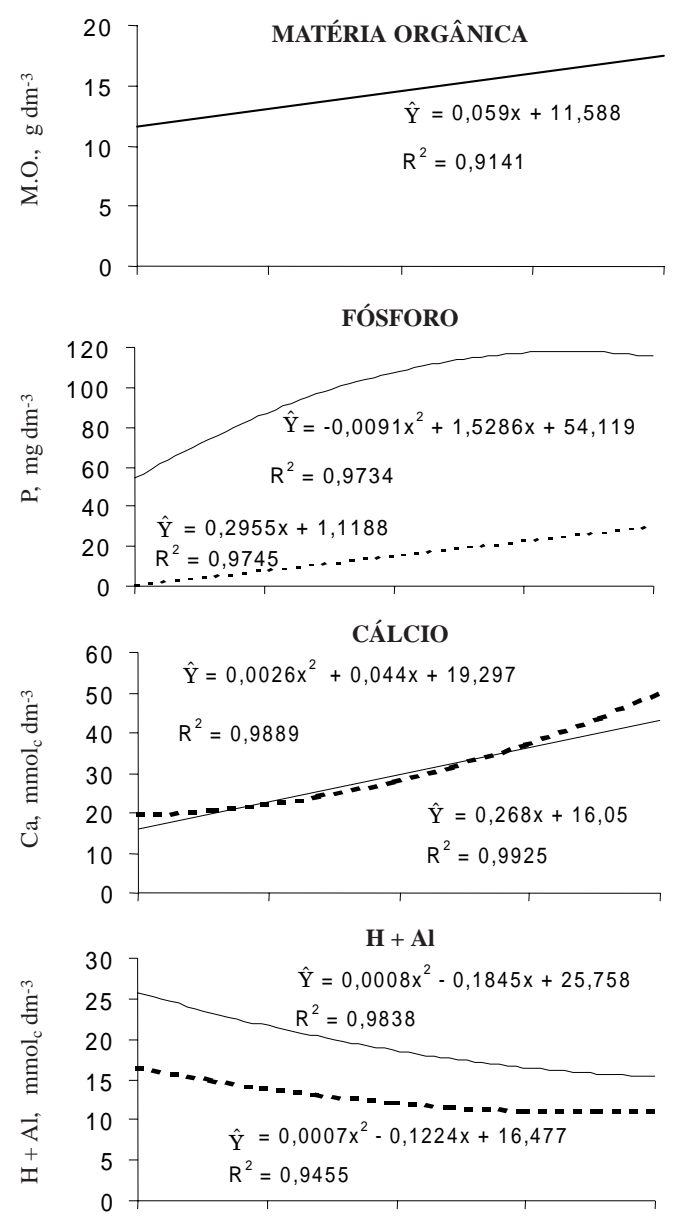

CTC

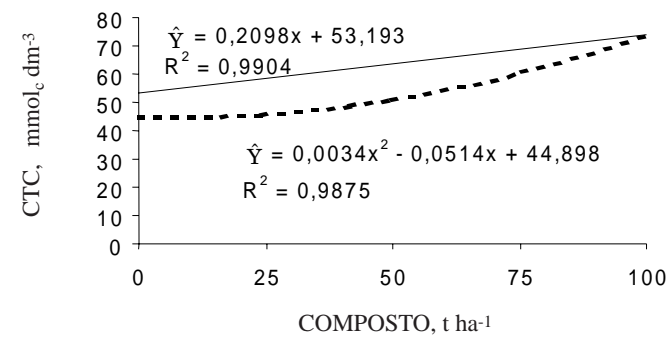

Os aumentos de P, K, Ca e Mg obtidos em função de doses do composto (Figura 1) provavelmente sejam devidos à mineralização de parte desses el ementos que estavam na forma orgânica. No caso do $\mathrm{P}$, por exemplo, $100 \mathrm{t}$ ha-1 do composto com $5 \mathrm{~g} \mathrm{~kg}^{-1}$ de $\mathrm{P}_{2} \mathrm{O}_{5}$ correspondem a $500 \mathrm{~kg} \mathrm{ha}-1$ de $\mathrm{P}_{2} \mathrm{O}_{5}$. Ayuso (1991), citado por Costa et al. (1992), observou incrementos nos teores de $\mathrm{P}$ assimilável devidos ao efeito residual do composto no solo. Esses resultados estão de acordo com os obtidos por Mazur et al. (1983), em Latossolo Amarel o textura arenosa, com a cultura do milho; segundo os quais os fenômenos de redução de fixação de fósforo e de mineralização
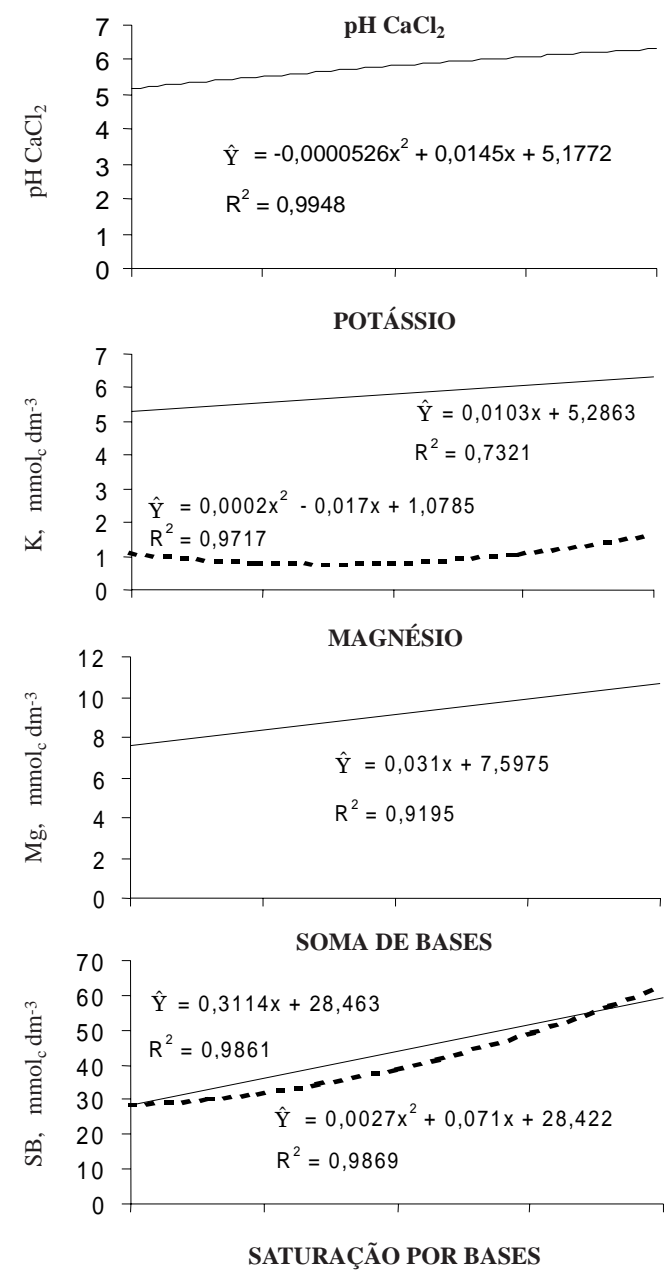

Figura 1. Efeito do composto de lixo urbano, na ausência (- - -) e na presença (-) de adubação mineral nas propriedades químicas do solo, variáveis com apenas uma regressão indicam ausência de interação significativa. 
de matéria orgânica constituem possíveis causas dos maiores níveis de P assimilável no solo.

$\mathrm{O}$ teor de $\mathrm{H}+\mathrm{Al}$ (Figura 1 ) diminuiu com o aumento da dose do composto, tanto na presença quanto na ausência de adubação mineral, enquanto a soma de bases e o pH, como era esperado, aumentaram. É provável que os mai ores valores de $\mathrm{H}+\mathrm{Al}$ na presença de adubação mineral sejam devidos ao caráter acidificante das soluções utilizadas como fontes de nutrientes. A el evação do val or de pH dosolo está deacordo com as observações de diversos autores de que, de maneira geral, a aplicação de composto humificado ao solo promove elevação do seu pH (Coker \& Matthews, 1983; Simeoni et al., 1984; Pereira Neto, 1987). Um fator importante que pode ter colaborado para a diminuição da acidez potencial éa possível formação de complexos estáveis entreo Al trocável ea matéria orgânica aplicada (Stevenson, 1982).

A aplicação do composto promoveu el evação dos teores de matéria orgânica do solo, o que teria aumentado também a CTC (Figura 1). A saturação por bases também aumentou com as doses do composto, sendo os val ores de V\% mais el evados na ausência de adubo mineral, talvez em razão dos menores teores de $\mathrm{H}+\mathrm{Al}$ nestas condições.

\section{Teores totais de Fe, Mn, $\mathrm{Zn}$ e Ni no solo}

Os teores totais dos micronutrientes $\mathrm{Fe}, \mathrm{Mn}$ e $\mathrm{Zn}$ aumentaram em função das doses aplicadas do composto (Quadro 1), tendo sido os maiores incrementos observados para o Zn. Purves \& Mackenzie (1973) relataram aumento nos teores de Zn no solo em níveis excessivos após a aplicação de 100 t ha-1 $^{-1}$ de composto. Aumentos significativos no conteúdo total desse elemento também foram relatados por Cabrera et al. (1989) com o emprego de 14 t ha-1 de composto. Segundo Chander \& Brookes (1991), teores muito el evados de $Z n$ causaram redução significativa na decomposição da matéria orgânica do sol o e decréscimo na quantidade de biomassa mi crobiana; contudo, oteor considerado muito al to foi de $281 \mathrm{mg} \mathrm{kg}^{-1}$, valor muito acima do observado neste experimento.

Baseando em dados da Environmental Protection Agency (1983), que considera como teores totais máximos permitidos no solo em mg kg-1: 1.000, 500 e 100, respectivamente, para $\mathrm{Zn}, \mathrm{Mn}$ e $\mathrm{Ni}$, e de autores citados por Egreja Filho (1993), pode-se inferir que, apesar de os teores totais desses elementos terem aumentado com a adição do composto, ainda se mostraram abaixo daqueles considerados excessivos. Embora não seja um bom

Quadro 1. E feito do composto de lixo urbano nos teores totais e disponíveis de micronutrientes e metais pesados do solo, na ausência e na presença de adubação mineral

\begin{tabular}{|c|c|c|c|c|c|c|c|c|}
\hline \multirow{2}{*}{ Composto } & \multicolumn{4}{|c|}{ Teores totais } & \multicolumn{4}{|c|}{ Teores disponíveis $^{(1)}$} \\
\hline & $\mathbf{F e}$ & Mn & $\mathrm{Zn}$ & $\mathbf{N i}$ & $\mathbf{F e}$ & Mn & $\mathrm{Zn}$ & $\mathbf{N i}$ \\
\hline \multirow[t]{2}{*}{$\mathrm{t} \mathrm{ha}^{-1}$} & \multicolumn{8}{|c|}{$-\mathrm{mg} \mathrm{kg}^{-1}$} \\
\hline & \multicolumn{8}{|c|}{ Ausência de adubação mineral ${ }^{(2)}$} \\
\hline 0 & 9.510 & 61,0 & 6,8 & 20,3 & 8,4 & 5,0 & 0,4 & 0,1 \\
\hline 12,5 & 9.493 & 59,5 & 10,0 & 23,3 & 8,4 & 4,7 & 0,8 & 0,1 \\
\hline 25,0 & 9.543 & 60,5 & 12,3 & 22,8 & 7,9 & 4,2 & 1,2 & 0,1 \\
\hline 50,0 & 9.560 & 60,5 & 13,3 & 29,0 & 7,9 & 3,9 & 1,9 & 0,2 \\
\hline \multirow[t]{2}{*}{100,0} & 9.673 & 64,0 & 17,0 & 33,3 & 9,9 & 4,0 & 3,7 & 0,2 \\
\hline & \multicolumn{8}{|c|}{ Presença de adubação mineral ${ }^{(2)}$} \\
\hline 0 & 9.573 & 64,8 & 9,0 & 14,8 & 31,2 & 23,2 & 1,9 & 0,1 \\
\hline 12,5 & 9.580 & 68,3 & 14,8 & 17,8 & 26,0 & 22,3 & 2,8 & 0,1 \\
\hline 25,0 & 9.600 & 65,8 & 11,8 & 14,0 & 23,3 & 17,3 & 2,8 & 0,2 \\
\hline 50,0 & 9.748 & 67,3 & 14,0 & 21,8 & 22,4 & 12,7 & 3,8 & 0,2 \\
\hline \multirow[t]{2}{*}{100,0} & 10.078 & 73,3 & 20,8 & 16,3 & 21,9 & 10,3 & 5,2 & 0,3 \\
\hline & \multicolumn{8}{|c|}{$\mathbf{F}$} \\
\hline$A^{(3)}$ & $12,9 *$ & $113,2^{*}$ & $3,3^{*}$ & $49,4^{*}$ & $1.620,9^{*}$ & $1.928,8^{*}$ & $407,1^{*}$ & $11,3^{*}$ \\
\hline C & $8,1^{*}$ & $11,1^{*}$ & $8,5^{*}$ & $6,7^{*}$ & $16,7 *$ & $87,9 *$ & $178,0^{*}$ & $48,2^{*}$ \\
\hline$A \times C$ & $2,2^{\text {ns }}$ & $2,7^{\text {ns }}$ & $0,6^{\text {ns }}$ & $2,9 *$ & $19,5^{*}$ & $65,8^{*}$ & $1,9^{\text {ns }}$ & $5,1 *$ \\
\hline C.V. (\%) & 1,5 & 3,1 & 29,7 & 18,6 & 7,7 & 8,6 & 10,9 & 12,5 \\
\hline
\end{tabular}


critério para avaliar a contaminação do solo com metais pesados, os teores totais tềm sido os mais utilizados, uma vez que ainda não foram selecionados extratores adequados para a determinação dos teores disponíveis para as plantas.

Deve-se observar, principal mente no caso do Zn, que se pode atingir o teor total máximo tolerável no solo (50 mg kg-1, segundo Lindsay, citado por Egreja Filho, 1993), após três ou quatro anos de aplicações

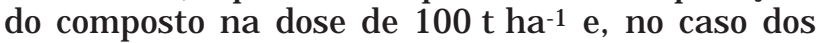
outros micronutrientes, prazos maiores seriam necessários. Por isso, o monitoramento dos teores de micronutrientes no composto e das doses aplicadas é importante para prevenir a contaminação do solo.

Os teores totais de $\mathrm{Ni}$ aumentaram linearmente ( $\hat{Y}=20,8063+0,1305 x, R^{2}=0,95$ ) apenas na ausência de adubo mineral e estão na faixa considerada comum para solos (abaixo de $40 \mathrm{mg} \mathrm{kg}^{-1}$ ), segundo Lindsay (1979), citado por Egreja Filho (1993).

\section{Teores de Fe, Mn, Zn e Ni extraídos com DTPA no solo}

O conteúdo de Zn extraído com DTPA aumentou em função das doses de composto, e os de Mn e Fe diminuíram na presença de adubo mineral e não se alteraram na sua ausência (Quadro 1). Aumentos na quantidade de $\mathrm{Zn}$ e Ni extraídos com DTPA foram observados por Street et al. (1977) e Schauer et al. (1980), citados por Petruzzelli et al. (1989), como efeito da aplicação de lodo de esgoto ao solo.

É possível que a diminuição dos teores de $\mathrm{Mn}$ e Feextraídos com DTPA com a aplicação do composto e na presença de adubo mineral se deva à formação de complexos muito estáveis desses metais com a matéria orgânica (Borkert, 1991). Essa diminuição não foi observada na ausência de adubo mineral, provavelmente pelos menores teores de $\mathrm{Mn}$ e Fe existentes nestas condições.

Houve elevação do conteúdo de Ni extraído com DTPA em função de doses aplicadas de composto, tanto na ausência como na presença de adubo mineral; no entanto, as quantidades extraídas foram muito menores que os teores totais e não causaram fitotoxidez nas plantas.

Observando os dados do quadro 1 , constata-seque o aumento no conteúdo total dos micronutrientes não se refletiu na quantidade extraída com DTPA, exceto para o Zn.

\section{Perfilhamento e produção de matéria seca}

O perfilhamento do sorgo foi influenciado apenas pela adubação mineral, não sendo afetado por doses de composto. $\mathrm{Na}$ presença de adubo mineral, as plantas produziram de três a quatro perfilhos e, em sua ausência, não perfilharam. A produção de matéria seca de sorgo aumentou com a dose de composto, porém, esseefeitofoi significativo somente na ausência de adubação mineral (Figura 2). Apesar de o composto ter aumentado a produção de matéria seca na parte aérea, seu efeito foi menor que o da adubação mineral, concordando com resultados de Ryan et al. (1985), citados por Costa et al. (1992), que verificaram menor eficiência do composto em comparação à fertilização mineral com N, P e K em cultivo de ciclo curto.

A menor eficiência do composto pode ser explicada pelo fato de a maior proporção de $\mathrm{N}$ e $\mathrm{P}$ nele contido estar na forma orgânica, necessitando de mineral ização para que esses nutrientes possam ser utilizados pelas plantas (Costa et al., 1992). Além disso, os baixos teores de $K$ no composto também podem ter limitado o crescimento das plantas. Devese atentar para o fato de que os aumentos expressivos de nutrientes no sol o em função de doses do composto (F igura 1), principal mente de $P$ resina, foram observados no final do experimento, ou seja, aproximadamente 140 dias após a adição do composto. Esse tempo permitiu a mineralização de parte do $P$ orgânico aplicado, o que não significa que a quanti dadeliberada tenha atendidoàs necessidades das plantas. O mesmo fato deve ter ocorrido com o $\mathrm{N}$, levando a um menor desenvol vimento das plantas. Ao utilizar o composto, deve-se levar em conta a necessidade de um período suficientemente longo, para que ocorra a mineralização dos nutrientes, ou de uma freqüência de aplicação, que el eve seu efeito residual.

\section{Quantidade acumulada de P, K, Ca, Mg, Fe, Mn, Zn e Ni nas plantas de sorgo}

As quantidades acumuladas de $\mathrm{K}, \mathrm{Ca}, \mathrm{Mg}$ e Zn nas plantas aumentaram em função de doses do composto, independentemente da presença ou não de adubação mineral (Quadro 2). A quantidade acumulada de $\mathrm{P}$ não foi afetada pelas doses do composto, e as de Fee Mn aumentaram na ausência de adubação mineral ediminuíram na sua presença.

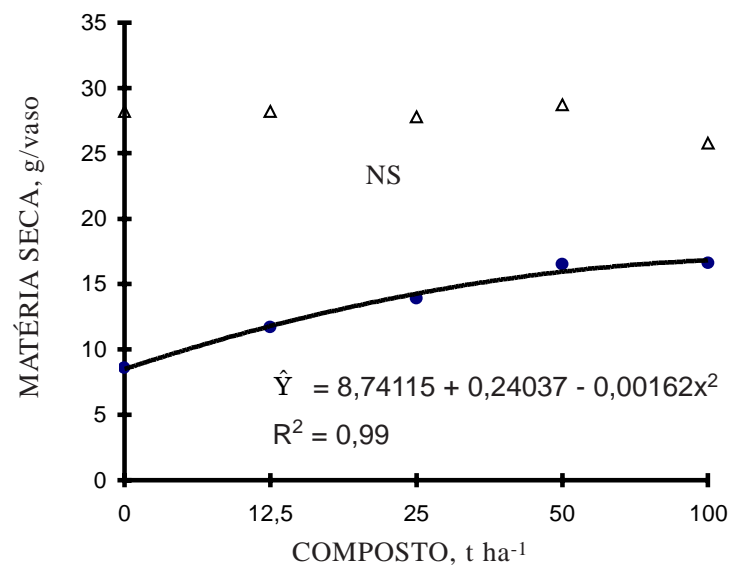

Figura 2. Produção de matéria seca de plantas de sorgo em função de doses do composto de lixo urbano, na presença $(\Delta)$ e na ausência $(\bullet)$ de adubação mineral. 
Quadro 2. Efeito do composto de lixo urbano na quantidade acumulada de macro e micronutrientes da parte aérea de plantas de sorgo, na ausência e na presença de adubação mineral

\begin{tabular}{|c|c|c|c|c|c|c|c|}
\hline Composto & $\mathbf{P}$ & $\mathbf{K}$ & $\mathbf{C a}$ & Mg & $\mathbf{F e}$ & Mn & $\mathbf{Z n}$ \\
\hline \multicolumn{8}{|c|}{ _ mg/vaso } \\
\hline & \multicolumn{7}{|c|}{ Ausência de adubação mineral(1) } \\
\hline 0 & 2,3 & 120,4 & 38,6 & 25,4 & 1,86 & 0,83 & 0,38 \\
\hline 12,5 & 2,3 & 157,7 & 59,2 & 33,5 & 2,41 & 1,03 & 0,43 \\
\hline 25,0 & 3,4 & 178,4 & 73,5 & 38,9 & 2,92 & 1,29 & 0,61 \\
\hline 50,0 & 5,8 & 228,3 & 80,8 & 38,1 & 3,41 & 1,49 & 0,66 \\
\hline \multirow[t]{2}{*}{100,0} & 3,6 & 326,7 & 101,1 & 34,8 & 4,53 & 1,75 & 0,81 \\
\hline & \multicolumn{7}{|c|}{ Presença de adubação mineral ${ }^{(1)}$} \\
\hline 0 & 35,2 & 573,0 & 138,1 & 64,4 & 9,84 & 15,90 & 1,24 \\
\hline 12,5 & 34,4 & 584,3 & 156,4 & 71,4 & 7,91 & 17,65 & 1,43 \\
\hline 25,0 & 33,4 & 584,3 & 181,5 & 73,1 & 8,37 & 15,32 & 1,60 \\
\hline 50,0 & 33,4 & 668,1 & 199,1 & 76,0 & 7,36 & 12,52 & 1,90 \\
\hline \multirow[t]{2}{*}{100,0} & 31,0 & 614,7 & 213,2 & 69,3 & 5,83 & 9,06 & 2,07 \\
\hline & \multicolumn{7}{|c|}{$\mathbf{F}$} \\
\hline$A^{(2)}$ & $965,94 * *$ & $447,22 * *$ & $427,98 * *$ & $396,38 * *$ & $146,54 * *$ & $579,72 * *$ & $316,02 * *$ \\
\hline C & $0,58^{\mathrm{ns}}$ & $6,23 * *$ & $21,67 * *$ & $5,07^{* *}$ & $0,45^{\mathrm{ns}}$ & $6,52 * *$ & $14,13^{* *}$ \\
\hline$A \times C$ & $1,36^{\mathrm{ns}}$ & $2,43^{\mathrm{ns}}$ & $0,57^{\mathrm{ns}}$ & $0,26^{\mathrm{ns}}$ & $7,51^{* *}$ & $9,64 * *$ & $1,69^{\text {ns }}$ \\
\hline C.V. (\%) & 16,56 & 14,91 & 13,17 & 11,40 & 23,20 & 21,90 & 17,13 \\
\hline
\end{tabular}

(1) Adubação mineral realizada com solução nutritiva. ${ }^{(2)}$ Significância pelo teste $F$ : ** e ${ }^{\text {ns }}$ significativo a $1 \%$ e ns não-significativo, respecitvamente.

$A=$ fator adubação mineral; $C$ = fator dose de composto e $A X C=$ interação dos fatores.

As quantidades acumuladas de $\mathrm{Fe}, \mathrm{Mn}$ e $\mathrm{Zn}$ na planta acompanharam os teores extraídos do solo com solução de DTPA (Quadro 1), pois houve diminuição das quantidades de $\mathrm{Fe}$ e de $\mathrm{Mn}$ na presença de adubação mineral (apesar deter havido aumento nos teores totais desses el ementos no solo) e aumento da quantidade de Zn em função de doses de composto, com as plantas, provavel mente tendo mais $\mathrm{Zn}$ e menos Fe e $\mathrm{Mn}$ à disposição para serem absorvidos. Trindade et al. (1996) obtiveram resultados semelhantes, constatando menor absorção de $\mathrm{Mn}$ pelas plantas adubadas com composto de lixo, ao mesmo tempo em que o teor total desse el emento no sol o havia aumentado.

Segundo Dechen et al. (1991), a variação no conteúdo de micronutrientes das plantas é grande por causa da influência de diversos fatores, sendo os val ores geral mente encontrados na matéria seca da ordem, em mg kg-1, de 20 a 50 para o Zn; de 10 a 20, para o Mn, eem torno de 100, para oF e. Comparando esses valores com os obtidos no experimento, os quais, convertidos para $\mathrm{mg} \mathrm{kg}^{-1}$, apresentaram variação de 40 a 92, para o Zn; de 96 a 719, para o $\mathrm{Mn}$, e de 220 a 413, para o Fe, verificou-se que eles foram mais el evados do que os citados por aqueles autores.
O niquel não foi detectado na parte aérea das plantas, tanto na ausência como na presença de adubação mineral, e mesmo na dose mais elevada do composto.

\section{CONCLUSÕES}

1. O composto aumentou a produção de matéria seca do sorgo na ausência de adubação mineral; esta, contudo, promoveu maior aumento de produção e perfilhamento das plantas.

2. Na parte aérea do sorgo não foi detectada a presença de $\mathrm{Ni}$ e a quantidade acumulada dos micronutrientes $\mathrm{Fe}, \mathrm{Mn}$ e $\mathrm{Zn}$ aumentou com as doses do composto, na ausência de adubo mineral .

3. A aplicação do composto de lixo promoveu aumento dos val ores de $\mathrm{pH}$, matéria orgânica, bases trocáveis, $\mathrm{P}$ resina eCTC do solo, reduzindo a acidez potencial $(\mathrm{H}+\mathrm{Al})$.

4. Os teores totais deFe, Mn eZn no solorelacionaram-se significativamente e positivamente com as doses de composto aplicadas, mas não atingiram níveis considerados excessivos. O teor total de $\mathrm{Ni}$ foi influenciado apenas na ausência de adubo mineral . 
5. Os teores de $\mathrm{Zn}$ e $\mathrm{Ni}$ extraídos com DTPA aumentaram com as doses do composto, enquanto os de $\mathrm{Mn}$ e Fe não foram alterados na ausência de adubo mineral e diminuíram na sua presença.

\section{LITERATURA CITADA}

ALVES, W.L. Compostagem evermicompostagem notratamento de lixo urbano. 2.ed. J aboticabal, FUNEP, 1998. 53p.

BAKER, D.E. \& AMACHER, M.L. Nickel, copper, zinc and cadmium. 2.ed. In: PAGE, A.L.; MILLER, R.H. \& KEENEY, D.R., eds. Methods of soil analysis. Part 2. Madison, American Society of Agronomy, 1982. p.323-335.

BORKERT, C.M. Manganês. In: SIMPÓSIO SOBRE MICRONUTRIENTES NA AGRI CULTURA, 1., Piracicaba, POTAFOS/CNPq, 1991. p.173-189.

CABRERA, F .; DIAZ, E. \& MADRID, L. Effect of using urban compost as manure on soil contents of some nutrients and heavy metals. J . Sci. Food Agric., 47:159-169, 1989.

CHANDER, K. \& BROOKES, P.C. Effects of heavy metals from past applications of sewage sludge on microbial biomass and organic matter accumulation in a sandy loam and silty Ioam U.K. soil. Soil Biol. Biochem., 23:927-932, 1991.

COKER, E.G. \& MATTHEWS, P.J . Metals in sewage sludge and their potential effects in agriculture. Water Sci. Tech., 15:209-225, 1983.

COLLIER, L.S.; VELLOSO, A.C.X. \& AMARAL SOBRINHO, N.M.B. Distribuição de metais pesados em sol o gl ei tratado com composto de resíduo urbano. In: CONGRESSO BRASILEIRO DE CIÊNCIA DO SOLO, 26., Rio deJ aneiro, 1997. Resumo expandido... Rio de J aneiro, SBCS, 1997. CD-Rom

COSTA, F.; HERNANDEZ, T.; GARCIA, C.; AYUSO, M.; PASCUAL, J.A. \& APOLO, A. Efectoresidual de diferentes residuos orgánicos sobre um cultivo de cebada. Suelo Planta, 2:593-603, 1992.

COSTA, C.A.; CASALI, V.W.D.; LOURDES, E.G.; CECON, P.R. \& J ORDÃO, C.D. Teor de metais pesados em alface (Lactuca sativa L.) adubada com composto orgânico de lixo urbano. R. Ceres, 41:629-640, 1994.

DECHEN, A.R.; HAAG, H.P. \& CARMELLO, Q.A. Diagnose visual. In: FERREIRA, M.E. \& PESSOA DA CRUZ, M.C., eds. Micronutrientes na agricultura. In: SIMPÓSI O SOBRE MICRONUTRIENTES NA AGRI CULTURA, 1., Piracicaba, 1991. Anais. Piracicaba, POTAFOS/CNPq, 1991. p.273-288.

EGREJ A FILHO, F.B. Avaliação da ocorrência e distribuição química de metais pesados na compostagem do lixo domiciliar urbano. Viçosa, U niversidade F ederal deViçosa, 1993. 176p. (Tese de Mestrado)

ENVIRONMENTAL PROTECTION AGENCY. Hazardous waste land treatment. Cincinnati, 1983. p.198-281.

GROSSI, M.G.L. Avaliação da qualidade dos produtos obtidos de usinas de compostagem brasileiras de lixo doméstico através de determinação de metais pesados e substâncias orgânicas tóxicas. São Paulo, Universidade de São Paulo, 1993. 222p. (Tese de Doutorado)
HERNÁNDEZ, T.; GARCIA, C.; COSTA, F.; VALERO, J .A. \& AYUSO, M. Utilization of municipal waste as organic fertilizers. Suelo Planta, 2:373-383, 1992.

HERNANDO, S.; LOBO, M.C. \& POLO, A. Effect of theapplication of a municipal refuse compost on the physical and chemical properties of a soil. Sci. Total Environ., 81:589-596, 1989.

HORTENSTINE, C.C. \& ROTHWELL, D.F. Use of municipal compost in reclamation of phosphat-mining sand tailings. J . Environ. Qual., 1:415-418, 1972.

HORTENSTINE, C.C. \& ROTHWELL, D.F. Pelletized municipal refuse compost as a soil amendment and nutrient source for sorghum. J . Environ. Qual., 2:343-345, 1973.

J ARDIM, N.S.; WELLS, C.; PRANDINI, F.L.; ALMEIDA, M.L.O \& MANO, V.G.T. Lixo municipal: Manual degerenciamento integrado. São Paulo, IPT/CEMPRE, 1995. 278p.

KIEHL, E.J . Fertilizantes orgânicos. Piracicaba, CERES, 1985. 492p.

LINDSAY, W.L. \& NORWELL, W.A. Development of a DTPA soil test for zinc, iron, manganese and copper. Soil Sci. Soc. Am. J ., 42:421-428, 1978.

MAZUR, N.; SANTOS, G.A. \& VELLOSO, A.C.X. Efeito do composto de resíduo urbano na disponibilidade de fósforo em solo ácido. R. Bras. Ci. Solo, 7:153-156, 1983.

NAKAGAWA, J . Compostagem: obtenção e uso. In: GUERRINI, I.E. \& BULL, L.T., eds. ENCONTRO SOBRE MATÉRIA ORGÂNICA DO SOLO - problemas esoluções., 1., Botucatu, 1992. Anais. Botucatu, FEPAF, 1992. p.159-187.

PEREIRA NETO, J .T. On the treatment of municipal refuse and sewage sludge using aerated static pile composting: a low tecnology approach. Leeds, Universidade de Leeds, 1987. 276p. (Tese de Doutorado)

PETRUZZELLI , G.; LUBRANO, L. \& GUIDI, G. U ptake by corn and chemical extractability of heavy metals from a four year compost treated soil. Plant Soil, 116:23-27, 1989.

PURVES, D. \& MACKENZIE, J.E. Effects of applications of municipal compost on uptake of copper, zinc and boron by garden vegetables. Plant Soil, 39:361-371, 1973.

RAIJ , B. van; QUAGGIO, J .A.; CANTARELLA, H.; FERREIRA, M.E.; LOPES, A.S. \& BATAGLIA, O.C. Análise química do solo para fins de fertilidade. Campinas, Fundação Cargill, 1987. 170p.

SARRUGE, J .R. \& HAAG, H.P. Análises químicas em plantas. Piracicaba, ESALQ, 1974. 56p.

SIMEONI, L.A.; BARBARICK, K.A. \& SABEY, B.R. Effect of a small-scale composting of sewage sludge on heavy metal availability to plants. J . Environ. Qual., 13:264-268, 1984.

STEVENSON, F.J . Humus chemistry. New York, J ohn Wiley, 1982. 443p.

TRINDADE, A.V.; VILDOSO, C.I.A.; MUCHOVEJ, R.M.C. \& COSTA, L.M. I nteração de composto de lixo urbano e fungos micorrízicos na nutrição e crescimento do milho. R. Bras. Ci. Solo, 20:199-208, 1996. 